\title{
Clinical validation of free breathing Respiratory Triggered Retrospectively Cardiac Gated Cine Steady-State Free Precession (RT-SSFP) imaging in sedated children
}

\author{
Rajesh Krishnamurthy ${ }^{1,2^{*}}$, Amol Pednekar ${ }^{3}$, Esben Vogelius ${ }^{1}$, Lamya A Atweh $^{1}$, Zili D Chu' ${ }^{1}$ Wei Zhang ${ }^{1}$, \\ Prakash Masand', Shiraz A Maskatia², Shaine A Morris², Raja Muthupillai ${ }^{4}$
}

From 16th Annual SCMR Scientific Sessions

San Francisco, CA, USA. 31 January - 3 February 2013

\section{Background}

Cine steady-state free precession (SSFP) is the preferred sequence for ventricular function evaluation. However, SSFP demands uninterrupted RF excitation to maintain steady-state (SS) during suspended respiration. This is feasible in adults who can perform breath-holding (BH), but is difficult to accomplish in sedated or uncooperative children. To overcome this, many pediatric groups routinely perform multi-NSA acquisitions (MN) during free breathing. In this work, we validate a respiratory triggered (RT) SSFP sequence that drives the magnetization to steady-state before commencing cardiac gated cine acquisition in sedated pediatric population.

\section{Methods}

This prospective study was performed on 12 sedated children with congenital heart disease (age: $7 \pm 3$ yrs) with IRB approval.

\section{MRI Acquisition}

All imaging was performed on a commercial MR scanner (1.5 T, Achieva, Philips Healthcare). Identical imaging parameters were used for MN and RT cine SSFP sequences [1] covering both the ventricles in short-axis (SA) orientation (TR/TE/flip angle: $3 / 1.5 / 60$; acquired voxel size: $1.5-1.9$ x 1.5-2.1 x 7-8 mm3; SENSE acceleration factor: 2; temporal resolution: 30-45 ms; acquisition time: 8-10 RR intervals/slice).

${ }^{1}$ Pediatric Radiology, Texas Children's Hospital, Houston, TX, USA Full list of author information is available at the end of the article

\section{Data Analysis}

Image quality assessment (Figure 1) and quantitative volumetric analysis was performed on the datasets by independent blinded users. One-sided Wilcoxon signed rank test and Box plot analysis were performed to compare the RT-SSFP clinical scores against MN-SSFP. Bland-Altman (BA) analysis was performed on LV and RV volumes.

\section{Results}

The clinical scores for RT-SSFP were consistently better than MN-SSFP (Figure 1). BA analysis (Table. 1) indicates that variability between $\mathrm{RT}$ and $\mathrm{MN}$ acquisitions is comparable to inter and intra-observer variability reported in the literature [2]. In sedated children, the BMC $(\mathrm{p}=0.06)$ and EDef $(\mathrm{p}=0.06)$ scores were significantly better for RT-SSFP than MN-SSFP while ISA $(p=0.5)$ was comparable. The ISA due to inconsistent respiration level leads to inconsistent estimation of basal LV volume which can explain the variability in EDV and ESV using two different acquisitions. Total score (with equal weights to each clinical score category) was significantly better for RT compared to MN ( $\mathrm{p}=0.03)$. Total scan duration for SA stack using RT-SSFP $(4.1 \pm 0.97 \mathrm{~min})$ was slightly shorter than MN-SSFP $(4.6 \pm 0.9 \mathrm{~min})$ acquisitions.

\section{Conclusions}

The free breathing RT-SSFP sequence allows clinically diagnostic images in sedated children without any penalty for total scan time, and offers improved myocardial 


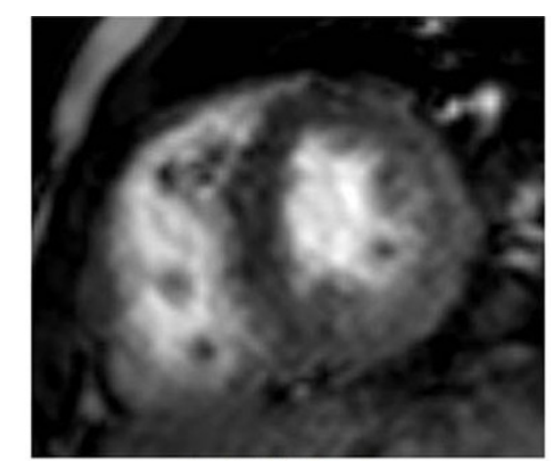

(A) EDef $=3$

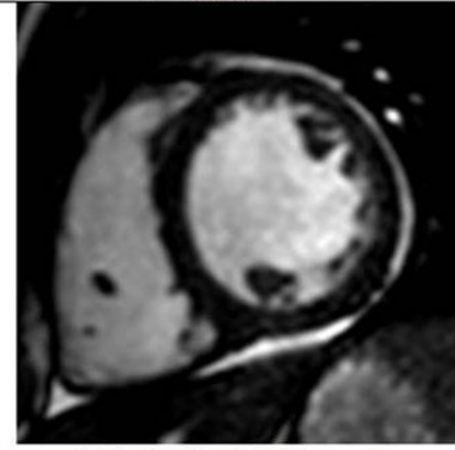

(D) $\mathrm{BMC} \& \mathrm{EDef}=1$

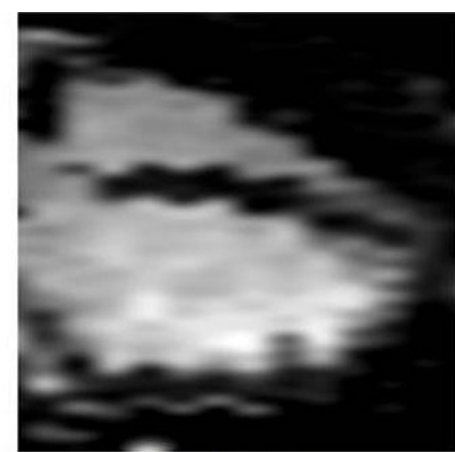

(B) ISA $=3$

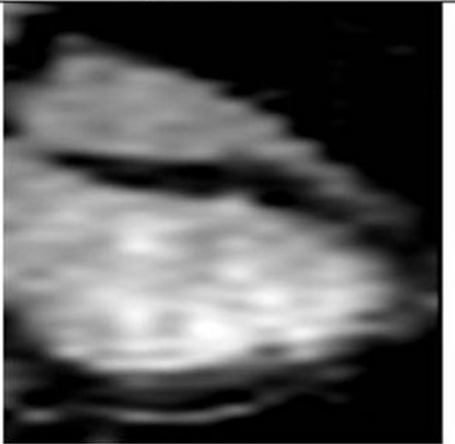

(E) ISA $=1$

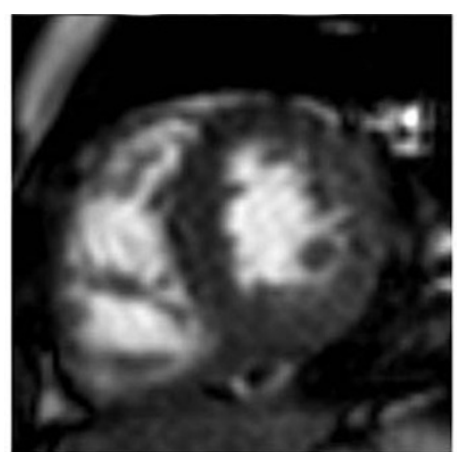

(C) $\mathrm{BMC}=2$

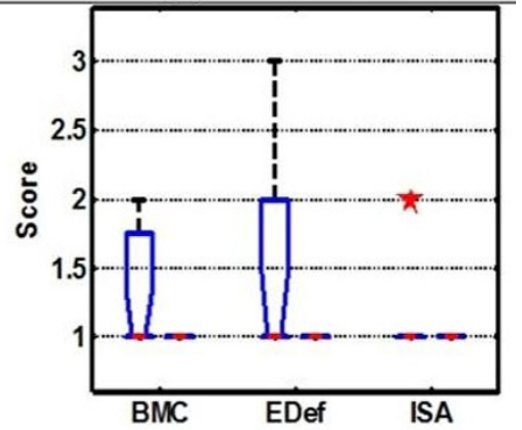

(F) Box plot (Left-MN, Right-RT)

Figure 1 (A-E) Representative images for the clinical scores. The clinical scoring system was as follows: A. Blood-myocardial contrast (BMC): 1-like SSFP, 2-like turbo GRE, 3-worse than turbo GRE; B. Endocardial edge definition (EDef): 1-excellent, 2-good, 3-poor; C. Inter-slice Alignment (ISA): 1Excellent alignment, $2-<2$ slices misaligned, 3- >2 slices misaligned. BMC score reflects quality of the steady state, and EDef score indicates intraslice motion blurring. ISA was determined by visualizing the SA stack as a volume. (F) Box plot for clinical scores depicts spread of the scores.

Table 1

\begin{tabular}{ccccccc}
\hline \multicolumn{7}{c}{ Table 1RT Vs. MN $(n=12)$} \\
\hline & EDV ml & ESV ml & EF \% & EDV ml & ESV ml & EF \% \\
\hline Bias & 4 & 2.2 & -0.8 & 3 & -0.4 & 1.1 \\
\hline Limit of Agreement & 8.6 & 6.6 & 8.2 & 17.5 & 10.8 & 9.1 \\
\hline
\end{tabular}

blood-pool contrast, and edge definition when compared to MN-SSFP.

\section{Funding}

None.

\section{Author details}

${ }^{1}$ Pediatric Radiology, Texas Children's Hospital, Houston, TX, USA.

${ }^{2}$ Departments of Pediatrics (Cardiology), Texas Children's Hospital, Houston, TX, USA. ${ }^{3}$ MR Research, Philips Healthcare, Houston, TX, USA. ${ }^{4}$ Department of Radiology, St. Luke's Episcopal Hospital and Texas Heart Institute, Houston, TX, USA.

Published: 30 January 2013

\section{doi:10.1186/1532-429X-15-S1-098}

Cite this article as: Krishnamurthy et al:: Clinical validation of free breathing Respiratory Triggered Retrospectively Cardiac Gated Cine Steady-State Free Precession (RT-SSFP) imaging in sedated children. Journal of Cardiovascular Magnetic Resonance 2013 15(Suppl 1):098.

\section{Submit your next manuscript to BioMed Central} and take full advantage of:

- Convenient online submission

- Thorough peer review

- No space constraints or color figure charges

- Immediate publication on acceptance

- Inclusion in PubMed, CAS, Scopus and Google Scholar

- Research which is freely available for redistribution 